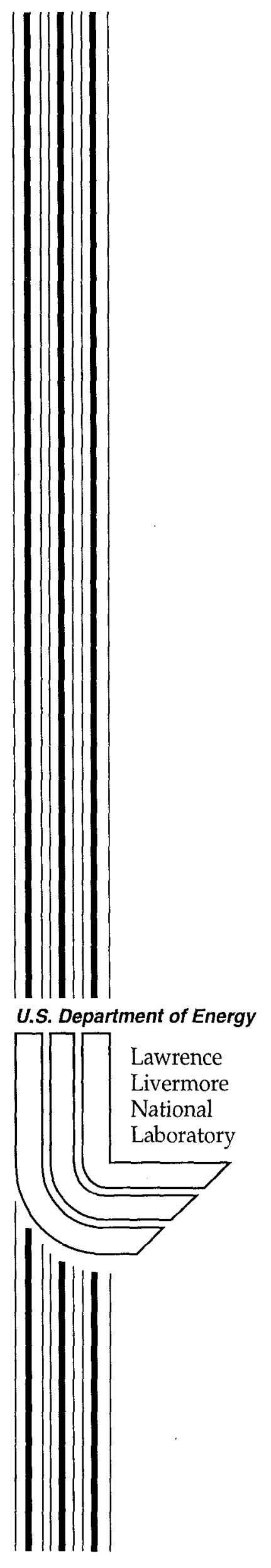

UCRL-ID-137802

\title{
The SSPX Bolometer Systems
}

K.I. Thomassen

February 2000 


\section{DISCLAIMER}

This document was prepared as an account of work sponsored by an agency of the United States Government. Neither the United States Government nor the University of California nor any of their employees, makes any warranty, express or implied, or assumes any legal liability or responsibility for the accuracy, completeness, or usefulness of any information, apparatus, product, or process disclosed, or represents that its use would not infringe privately owned rights. Reference herein to any specific commercial product, process, or service by trade name, trademark, manufacturer, or otherwise, does not necessarily constitute or imply its endorsement, recommendation, or favoring by the United States Government or the University of California. The views and opinions of authors expressed herein do not necessarily state or reflect those of the United States Goverrment or the University of California, and shall not be used for advertising or product endorsement purposes.

Work performed under the auspices of the U. S. Department of Energy by the University of California Lawrence Livermore National Laboratory under Contract W-7405-Eng-48.

This report has been reproduced directly from the best available copy.

Available to DOE and DOE contractors from the Office of Scientific and Technical Information

P.O. Box 62, Oak Ridge, TN 37831

Prices available from (423) 576-8401

http://apollo.osti.gov/bridge/

Available to the public from the

National Technical Information Service

U.S. Department of Commerce

5285 Port Royal Rd., Springficld, VA 22161

http://www.ntis.gov/

OR

Lawrence Livermore National Laboratory

Technical Information Department's Digital Library

http://www.llnl.gov/tid/Library.html 


\section{The SSPX Bolometer Systems}

\section{Total Power Bolometers}

There are two bolometry systems on SSPX, one that measures the total radiated power and a 16-channel array to measure the radiation profile. The first collimates the radiation through two slits in the horizontal plane spaced a distance $\mathrm{s}=1.2 \mathrm{~cm}$ apart as in Fig 1 . The slit heights are $h=1 / 100$ th of an inch, and the detector material is behind the second one. The number of electrons generated per photon is proportional to the photon energy (except for a factor of 3-4 enhancement in efficiency in the visible) so that the current of electrons is proportional to the power received. The power is in turn the product of the flux hitting the detector material and the projected perpendicular area of the slab material to the line of sight (which is often at an angle to the slab).

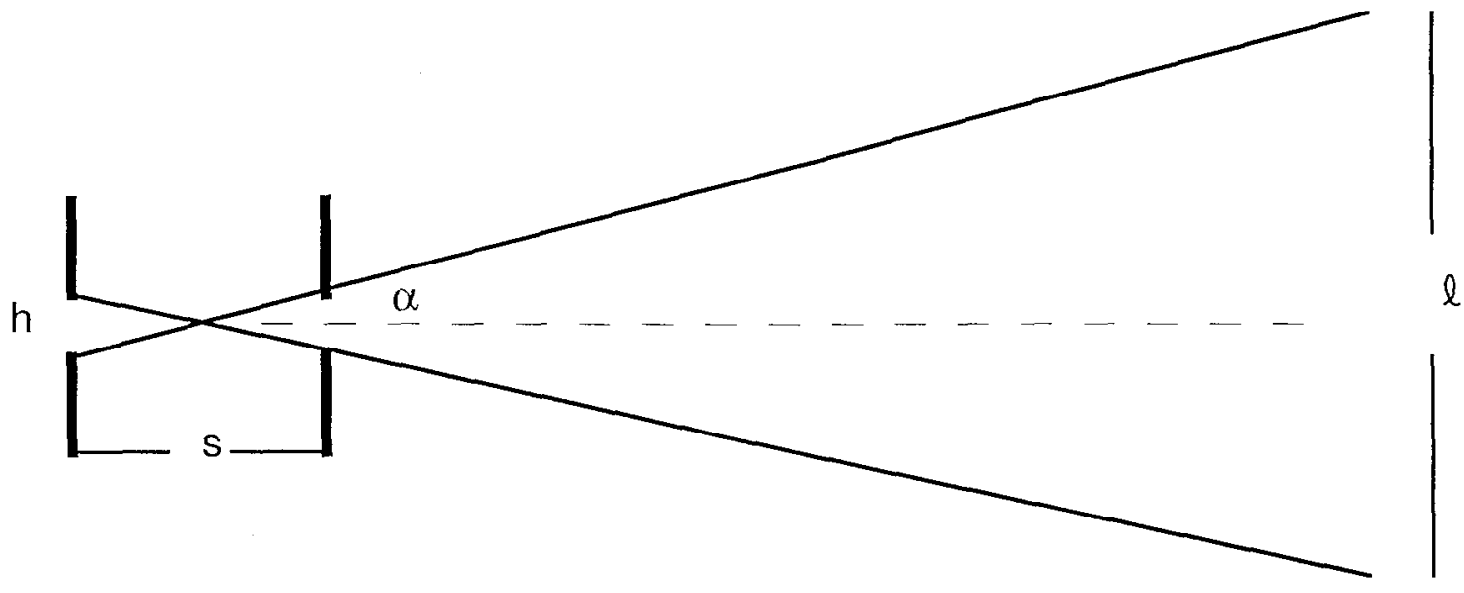

Fig. 1. Slit geometry defining the line-of-sight for the total power bolometers.

The manufacturers' specifications state that the bolometers generate 0.273 A/W of incident power. The current I is measured across a $50 \Omega$ resistor in a Camac 8210 , so that the bolometer power is

$$
P_{\text {det }}=\frac{\mathrm{I}}{0.273}=\frac{V}{13.65} \quad \text { so } \quad V=13.65 P_{\text {det }}
$$

The radiant flux $\mathrm{dF}\left(\mathrm{W} / \mathrm{cm}^{2}\right)$ from any given point in the plasma is $\frac{\mathrm{pdV}}{4 \pi \mathrm{r}^{2}}$, with $\mathrm{r}$ the distance between that point and the plasma. The integral $\mathrm{dV}$ is over all points in the plasma volume that can see some portion of the detector. Here, we assume $\mathrm{p}\left(\mathrm{W} / \mathrm{cm}^{3}\right)$ is a constant radiated power density.

For the total power bolometers (there are 3 of them) a perpendicular off the detector material passes through the center of the plasma. They are setback a distance $d=10 \mathrm{~cm}$ from the edge of the plasma of radius $a=50 \mathrm{~cm}$, and view a wedge of plasma that is $\ell \mathrm{cm}$ high (see Fig. 2) at the far edge of the plasma (a distance $2 \mathrm{a}+\mathrm{d}$ ). From the slit geometry (spacing $\mathrm{s}=1.2 \mathrm{~cm}$, height $\mathrm{h}=0.01 \mathrm{inch}$ ) 
the wedge is angled $\pm \alpha$ degrees from the horizontal, where $\alpha \approx \frac{h}{s}=\frac{0.0254}{1.2}$ (wedge angle of $\pm 1.2^{\circ}$ ). At the far edge of the plasma, at a distance $2 a+d$, the wedge height $l=2 \mathrm{hs}^{-1}(2 \mathrm{a}+\mathrm{d})$, so that $\ell=4.66 \mathrm{~cm}$. Using a laser source we measured $\ell \approx 5$, but its finite spot size over estimates the value.
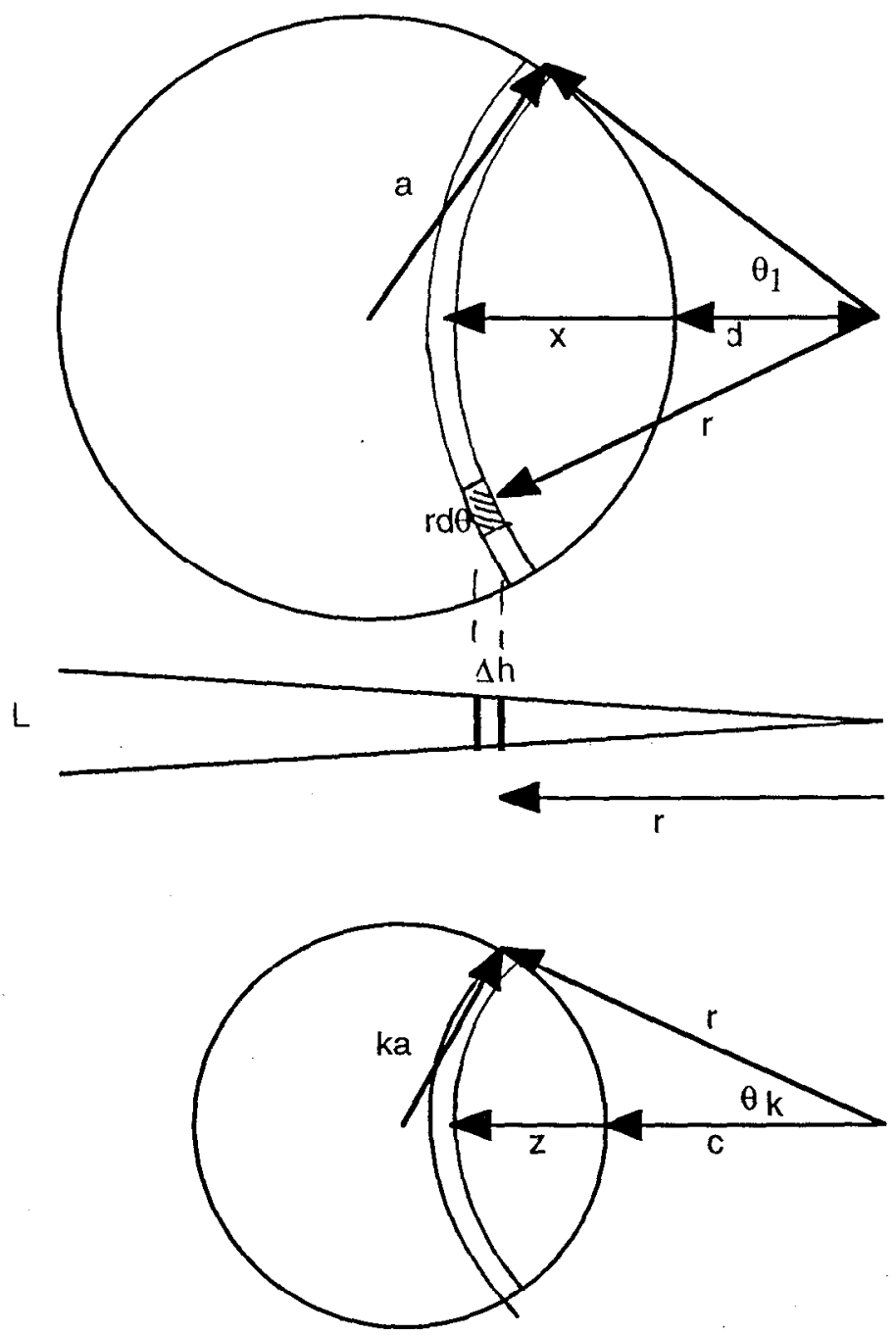

Fig. 2. Geometry of the view for total bolometry.

The detector area which multiplies the flux from different portions of the viewing wedge will vary with position in that wedge. For rays perpendicular to the detector and centered in the slots the full area $A_{\text {det }}$ is illuminated. $\quad\left(A_{d e t}=0.01 \mathrm{w} \mathrm{in}^{2}\right.$, with $w$ the detector width behind the slot). For rays above and below the midpane of the wedge the first slot shadows part of the second one, so that the area is decreased linearly over angles $\phi$ off that plane. For rays off to the sides of the wedge over angles $\theta$ the projected area of the detector is $A_{d e t} \cos \theta$, so that our integral is now 


$$
P_{d e t}=\int_{0}^{2 a} d x \frac{p}{4 \pi r^{2}} \int_{0}^{\alpha} 2 r d \phi \int_{0}^{\theta_{1}}\{A(\phi) \cos \theta\} 2 r d \theta
$$

Here, $\cos \theta_{1}=\frac{r^{2}+(a+d)^{2}-a^{2}}{2 r(a+d)}$ and $A(\phi)=A_{\operatorname{det}}\left(1-\frac{\phi}{\alpha}\right)$. Then,

$$
P_{\text {det }}=\frac{\alpha}{2 \pi} p A_{d e t} \int_{0}^{2 a} \sin \theta_{1} d x
$$

Let $x=a u, d=b a$, then

$$
P_{\text {det }}=\frac{h a}{2 s \pi} p A_{\text {det }} \int_{0}^{2} \sin \theta_{1} d u
$$

with $\quad \cos \theta_{1}=\frac{u(u+2 b)+2 b(1+b)}{2(u+b)(1+b)}$

For $b=1 / 5$, the integral is 1.457 so

$$
P_{\text {det }}=\frac{1.457 \text { ha }}{2 s \pi} p A_{\text {det }}=\frac{p A_{\text {det }}}{4.07}=\frac{V(\text { volts })}{13.65} \quad \text { watts }
$$

The radiated power from the plasma is $P_{\text {rad }}(M W)=\pi a^{2} L p$, so with $L=a=50$,

$$
P_{\text {rad }}(M W)=0.125 \pi p=\frac{V(\text { volts })}{8.54 A_{\operatorname{det}}\left(\mathrm{cm}^{2}\right)} \quad \text { MWatts }
$$

Two of the detectors are $1 / 4 \mathrm{~cm}$ wide (one bare, one covered with glass) while the third is $1 / 2 \mathrm{~cm}$ wide and covered with lithium flouride. For the smaller detectors the radiated power is $18.74 \mathrm{MW}$ per volt while it is half of that for the larger one.

If one assumes that the radiated power is constant over part of the plasma and zero elsewhere it is easy to show that one will calculate the same total power radiated (but higher power density where it does radiate). The voltage on the detector is a measure of the number of photons that get there times their energy. So it does not matter where (what profile) they come from, the total power will be the same. For example, in Fig 2 if we assume the power density is constant out to a radius $k a$, where $0<k<1$, we can repeat the calculation as before, except using

$$
\cos \theta_{k}=\frac{r^{2}+(a+d)^{2}-(k a)^{2}}{2 r(a+d)}
$$

Equating the measured voltage to the new value of $P_{\text {det }}$ will give a new value of $p$ over this limited region, but the total power radiated will be the same as can be shown by direct integration. 


\section{Radiation Profiles}

Radiation profiles are determined by a sixteen channel bolometer system shown here in Fig. 3. A collimating hole ahead of a detector array defines 16 different sight-lines through the plasma, the first ray passing through the center of the spheromak and the $16^{\text {th }}$ passing close to the edge. Each ray passes through a subset of the zones, each zone defined by the ray passing through it tangentially. So the first zone is the center of the plasma, the next annular region is zone 2, etc. The radial extent of each zone is variable and determined by the exact angular spread of the rays. At the tangency point, a ray bisects the zone, so the angle of the rays determines the width of a given zone. The angles in turn are defined by the geometry of the detector array.

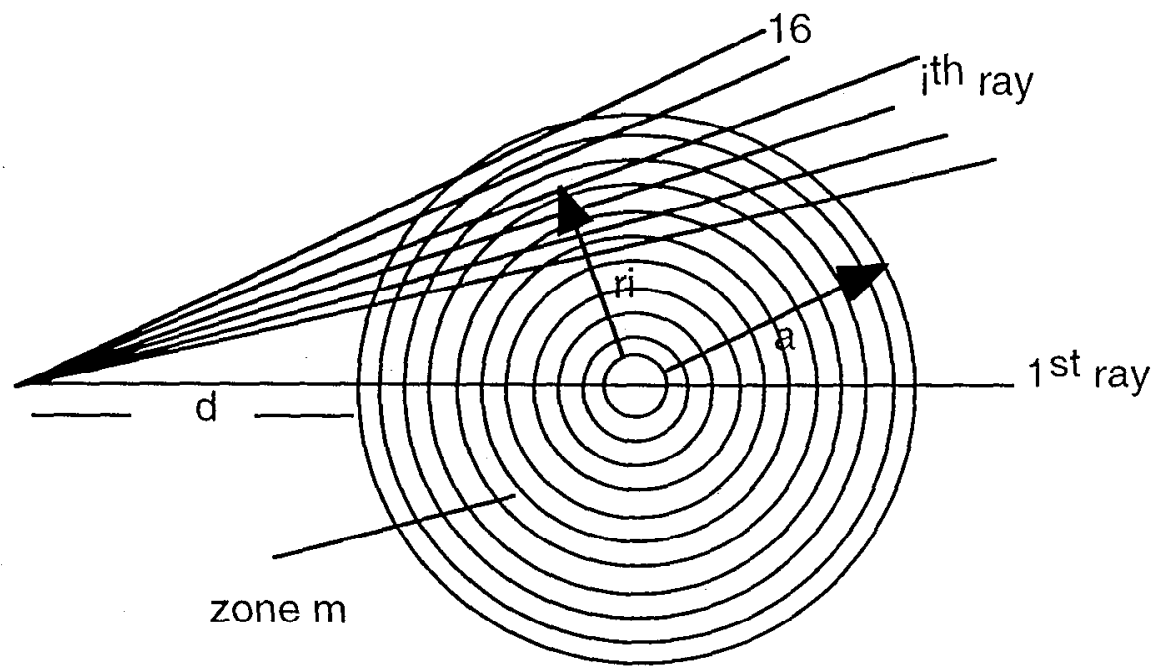

Fig 3. The ray and zone geometry for the SSPX array

The channels are defined by a circular pinhole in front of an array of 16 pinholes in a line. The 16 SSPX detectors are behind 0.02" diameter holes in a 0.01 " thick plate, and spaced 0.08 " apart. A second plate of the same thickness, parallel to it but $L \approx 1$ " away, has a $0.025^{\prime \prime}$ hole located such that the perpendicular from its center strikes the detector plate midway between the $8^{\text {th }}$ and $9^{\text {th }}$ channel and is pointed between the $8^{\text {th }}$ and $9^{\text {th }}$ ray through the plasma. The rays eminate from the 0.025 " hole which is $9.17 \mathrm{~cm}$ from the edge of the plasma. The collimating geometry is shown in Fig 4.

The angles $\alpha$ and $\beta$ are calculated from the geometry shown,

and

$$
L \tan \alpha_{n}=0.04+0.08 n-0.01-0.0125=0.0175+0.08 n
$$

Then

$$
(L+0.02) \tan \beta_{n}=0.04+0.08 n+0.01+0.0125=0.0625+0.08 n
$$

$$
\Delta \theta_{n}=\beta_{n}-\alpha_{n} \quad \text { and } \quad \theta_{n}=\alpha_{n}+0.5\left(\beta_{n}-\alpha_{n}\right)
$$


where $\theta_{\mathrm{n}}$ is the angle of the ray from the $\mathrm{n}^{\text {th }}$ hole.

If ray \#1 is to pass through the center of the plasma, and ray \#16 within a specified distance from the edge $(r=50 \mathrm{~cm})$, then the distance $L$ between plates is fixed by that requirement. Since the data from bolometry is most sensitive to the edge value of power (Abel inversion unfolds from the outside inward) we choose to make the last ray pass within $1 \mathrm{~cm}$ of the edge, so the distance $r_{16}$ in Fig 3 is $49 \mathrm{~cm}$. Since $d=9.17 \mathrm{~cm}$ the angle between rays 1 and 16 must be $\sin ^{-1}\{49 / 59.17\}=$ $55.9063^{\circ}$, and the angle $\theta_{7}$ should be half of that $\left(27.9532^{\circ}\right)$. From the above equations the distance between plates is then $L=1.1303^{\prime \prime}$. For this spacing, the angles for the holes $n=0-7$ are given in Table 1 .

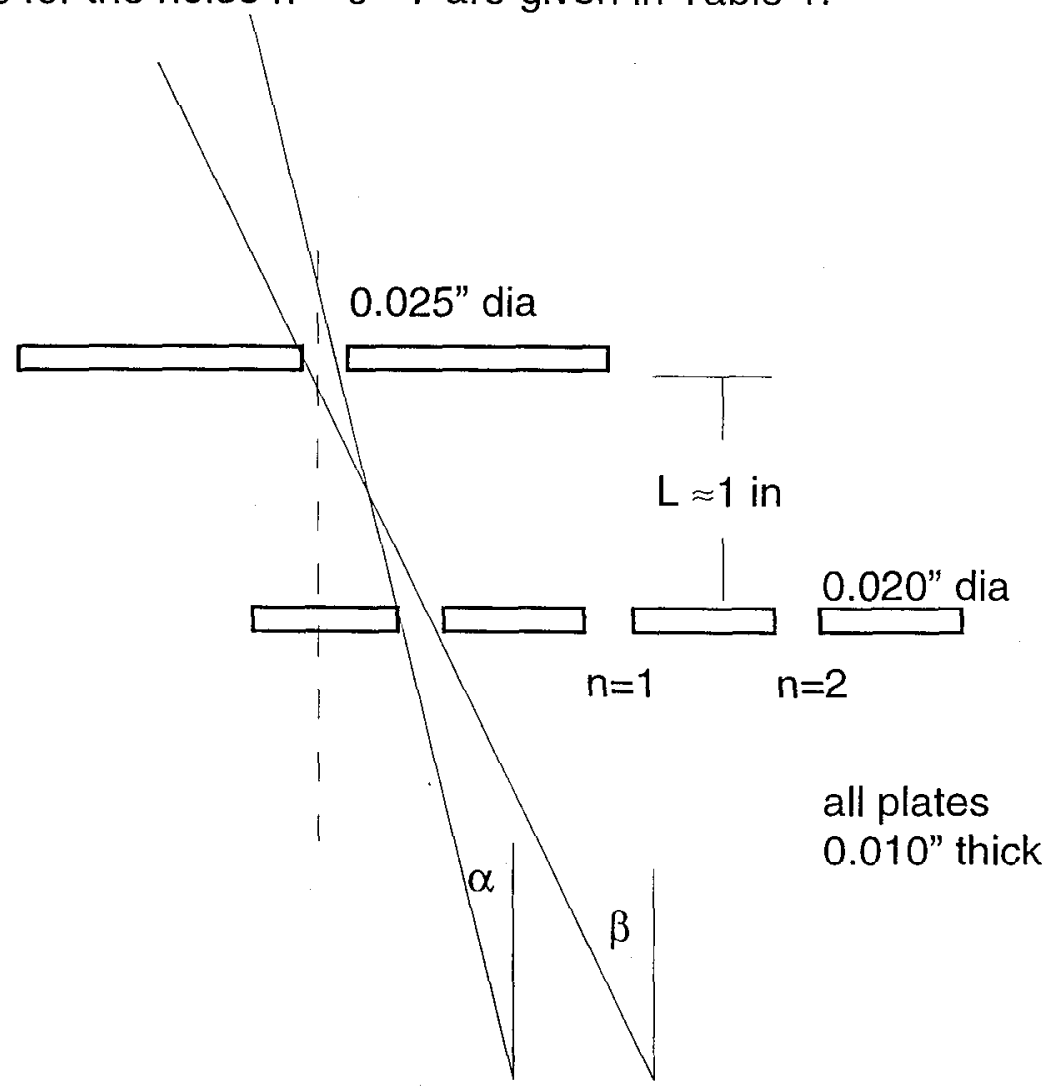

Fig. 4. Geometry of the detector channels.

Next, we want to know the length $\ell_{i}$ that the $i^{\text {th }}$ ray traverses between edges of the plasma. From the geometry in Fig. $5,\left(l_{i} / 2\right)^{2}=a^{2}-\left(r_{i}\right)^{2}$ and $r_{i}=(a+d) \sin \phi_{i}$. These length $\ell_{i}$ are the sum of the lengths of the $i^{\text {th }}$ ray through the $m$ radial zones it passes, $\ell_{i}=\sum \ell_{i-m}$. Here, $\phi_{1}=0^{\circ}$ and the remaining angles can be calculated using the values from Table 1 for the different holes. Table 2 gives the values of $r_{i}$, $\ell_{i}$, and $\phi_{i}$ for the 16 rays. We also compute the radii $\rho_{m}$ of the zone boundaries, by splitting the distance between adjacent rays, and the width of each zone. The center of the plasma is zone 1 and the outer radii are $\rho_{m}=r_{m}+\frac{r_{m+1}-r_{m}}{2}$, except that $\rho_{16}=50 \mathrm{~cm}$. 
Table 1

Angular spread of rays in SSPX

$\begin{array}{cccccc}\mathrm{n} & \mathrm{L} & \text { alpha } & \text { beta } & \text { theta } & (1 / \cos \theta)^{\wedge} 4 \\ & & & & & \\ 0 & 1.1303 & 0.887 & 3.165 & 2.026 & 1.001527 \\ 1 & 1.1303 & 4.93 & 7.185 & 6.058 & 1.016474 \\ 2 & 1.1303 & 8.925 & 11.14 & 10.03 & 1.052689 \\ 3 & 1.1303 & 12.83 & 14.98 & 13.91 & 1.110509 \\ 4 & 1.1303 & 16.63 & 18.7 & 17.66 & 1.19161 \\ 5 & 1.1303 & 20.27 & 22.25 & 21.26 & 1.298268 \\ 6 & 1.1303 & 23.76 & 25.64 & 24.7 & 1.433364 \\ 7 & 1.1303 & 27.06 & 28.84 & 27.95 & 1.600378\end{array}$

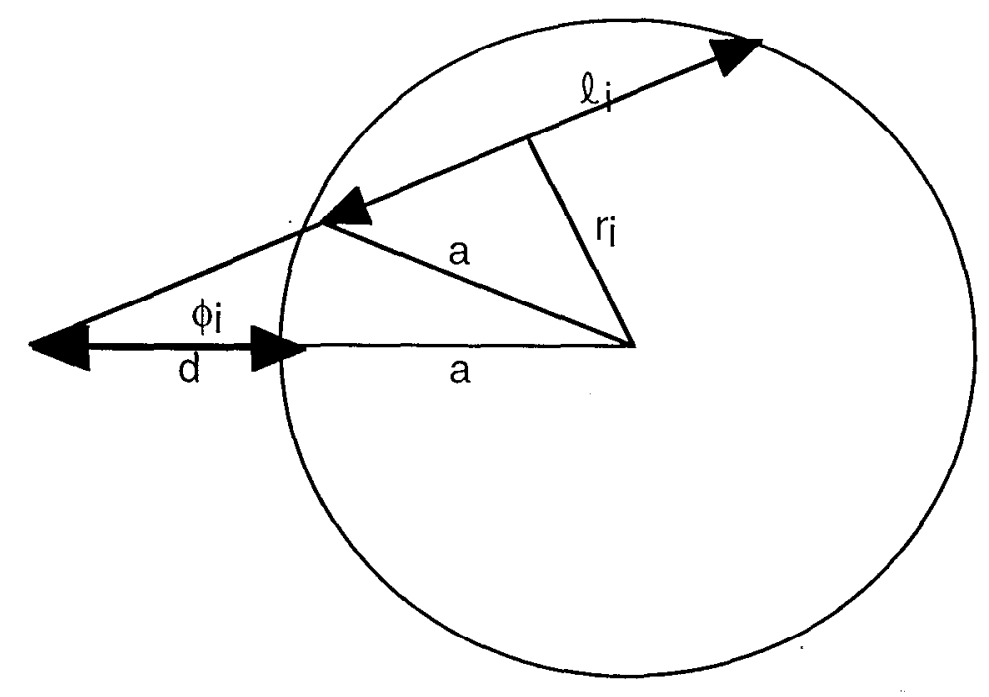

Fig. 5. Chord length for the ith ray.

Next we need the chord lengths for the $i$ th ray in each of the $m$ zones. Let $\ell_{i, m}$ be the length of the $i^{i \text { th }}$ ray through the $m^{\text {th }}$ zone (see Fig. 6 ). For $m<i$ the length is zero, and for $m=i$,

In the $m+1$ zone,

$$
\left(\ell_{i, m}\right)^{2}=4\left[\rho_{m}^{2}-\left(r_{i}\right)^{2}\right]
$$

$$
\ell_{i, m+1}=2 \sqrt{\left(\rho_{m+1}\right)^{2}-\left(r_{i}\right)^{2}}-2 \sqrt{\left(\rho_{m}\right)^{2}-\left(r_{i}\right)^{2}}
$$

Incrementing $m$ up to $m=16$ gives the remaining lengths. This proceedure then gives the $i^{\text {th }}$ row for the length matrix of Table 3 . The columns are the zones $m=1$ to 16 , and the total lengths sum correctly to those given in Table 2. 
Table 2

Angular spread of rays, and radii of rays and zones

$\begin{array}{cccccc}\text { ray } / & \phi & \text { ray } & \text { chord } & \text { zone } & \Delta \rho \\ \text { zone } & \text { angle } & \text { radius } & \text { length } & \begin{array}{c}\text { outer } \\ \text { radius }\end{array} & \text { width } \\ 1 & 0 & 0 & 100 & 1.68 & \\ 2 & 3.2556 & 3.36 & 99.77 & 5.127 & 3.447 \\ 3 & 6.6903 & 6.893 & 99.05 & 8.733 & 3.606 \\ 4 & 10.293 & 10.57 & 97.74 & 12.47 & 3.733 \\ 5 & 14.045 & 14.36 & 95.79 & 16.28 & 3.818 \\ 6 & 17.923 & 18.21 & 93.13 & 20.14 & 3.853 \\ 7 & 21.895 & 22.07 & 89.74 & 23.97 & 3.831 \\ 8 & 25.927 & 25.87 & 85.57 & 27.72 & 3.751 \\ 9 & 29.979 & 29.57 & 80.64 & 31.33 & 3.613 \\ 10 & 34.011 & 33.1 & 74.96 & 34.76 & 3.424 \\ 11 & 37.984 & 36.42 & 68.52 & 37.95 & 3.195 \\ 12 & 41.861 & 39.49 & 61.35 & 40.89 & 2.935 \\ 13 & 45.614 & 42.29 & 53.36 & 43.54 & 2.658 \\ 14 & 49.216 & 44.8 & 44.4 & 45.92 & 2.376 \\ 15 & 52.651 & 47.04 & 33.91 & 48.02 & 2.099 \\ 16 & 55.907 & 49 & 19.9 & 50 & 1.981\end{array}$

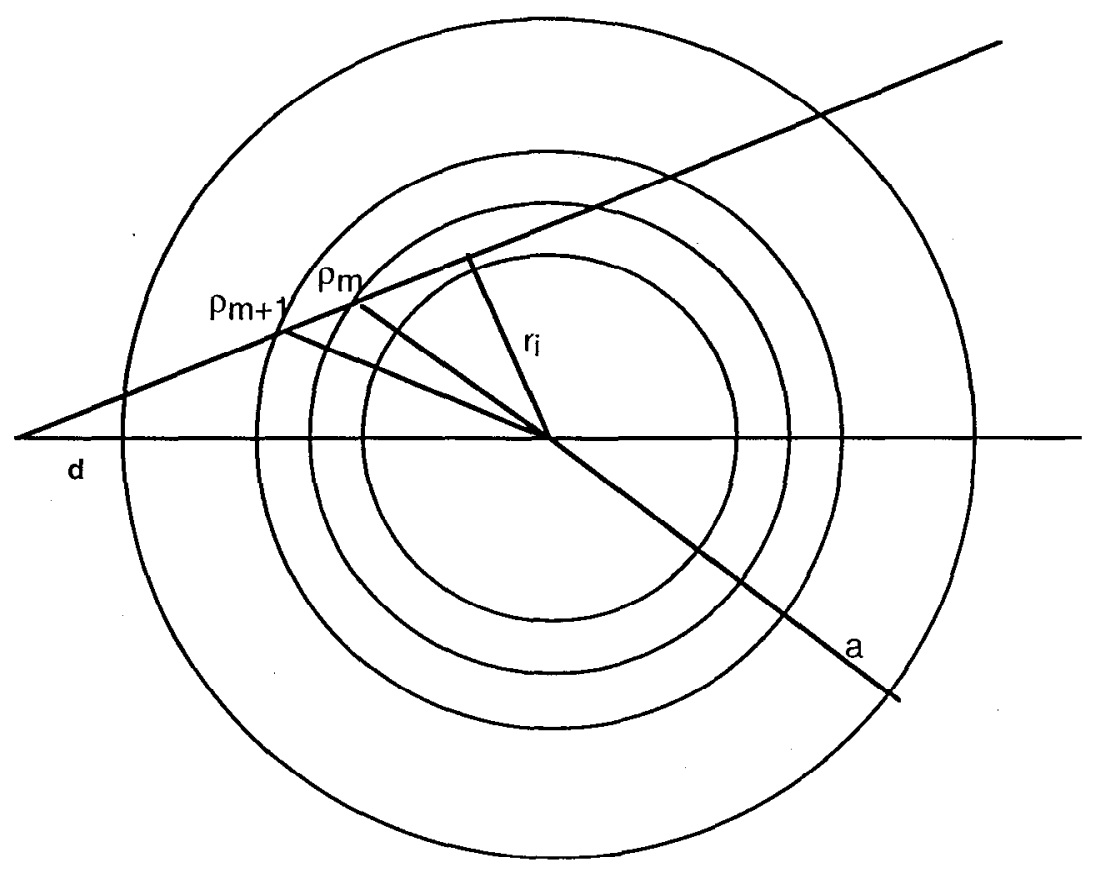

Fig. 6 Geometry for calculating length matrix elements. 
Table 3

Length matrix for the SSPX bolometer system

$\begin{array}{rrrrrrrrrrrrrrrr}3.36 & 6.89 & 7.21 & 7.47 & 7.64 & 7.71 & 7.66 & 7.5 & 7.23 & 6.85 & 6.39 & 5.87 & 5.32 & 4.75 & 4.2 & 3.96 \\ 0 & 7.74 & 8.38 & 7.89 & 7.86 & 7.84 & 7.75 & 7.57 & 7.27 & 6.88 & 6.42 & 5.89 & 5.33 & 4.77 & 4.21 & 3.97 \\ 0 & 0 & 10.7 & 10.1 & 8.73 & 8.33 & 8.07 & 7.78 & 7.43 & 7 & 6.51 & 5.96 & 5.39 & 4.81 & 4.24 & 4 \\ 0 & 0 & 0 & 13.2 & 11.6 & 9.51 & 8.74 & 8.23 & 7.74 & 7.23 & 6.68 & 6.09 & 5.49 & 4.89 & 4.31 & 4.06 \\ 0 & 0 & 0 & 0 & 15.4 & 12.9 & 10.1 & 9.04 & 8.28 & 7.61 & 6.96 & 6.3 & 5.65 & 5.02 & 4.41 & 4.14 \\ 0 & 0 & 0 & 0 & 0 & 17.2 & 14 & 10.6 & 9.2 & 8.21 & 7.39 & 6.62 & 5.89 & 5.2 & 4.55 & 4.27 \\ 0 & 0 & 0 & 0 & 0 & 0 & 18.7 & 14.8 & 10.9 & 9.22 & 8.05 & 7.09 & 6.24 & 5.46 & 4.76 & 4.44 \\ 0 & 0 & 0 & 0 & 0 & 0 & 0 & 19.9 & 15.4 & 11.1 & 9.11 & 7.79 & 6.73 & 5.83 & 5.03 & 4.67 \\ 0 & 0 & 0 & 0 & 0 & 0 & 0 & 0 & 20.7 & 15.8 & 11 & 8.89 & 7.46 & 6.34 & 5.4 & 4.97 \\ 0 & 0 & 0 & 0 & 0 & 0 & 0 & 0 & 0 & 21.2 & 15.9 & 10.9 & 8.58 & 7.07 & 5.92 & 5.37 \\ 0 & 0 & 0 & 0 & 0 & 0 & 0 & 0 & 0 & 0 & 21.4 & 15.8 & 10.6 & 8.2 & 6.65 & 5.92 \\ 0 & 0 & 0 & 0 & 0 & 0 & 0 & 0 & 0 & 0 & 0 & 21.2 & 15.5 & 10.2 & 7.77 & 6.7 \\ 0 & 0 & 0 & 0 & 0 & 0 & 0 & 0 & 0 & 0 & 0 & 0 & 20.8 & 15 & 9.7 & 7.86 \\ 0 & 0 & 0 & 0 & 0 & 0 & 0 & 0 & 0 & 0 & 0 & 0 & 0 & 20.1 & 14.4 & 9.84 \\ 0 & 0 & 0 & 0 & 0 & 0 & 0 & 0 & 0 & 0 & 0 & 0 & 0 & 0 & 19.3 & 14.6 \\ 0 & 0 & 0 & 0 & 0 & 0 & 0 & 0 & 0 & 0 & 0 & 0 & 0 & 0 & 0 & 19.9\end{array}$

The next task is to compute the power received in each of the 16 detectors, assuming the radiated power density is constant throughout a zone but different in each zone.

\section{Power on a bolometer array}

A bolometer array consists of a circular pinhole, of small but finite extent, on a plane (2) close to a second plane (3) with a linear array of bolometers centered along the line $y_{0}=0$. We want to calculate the power received on any one of the bolometer surfaces in that array. These two planes are separated by a distance $\ell_{0}$. We first map the radiation from a third plane (1) in the plasma at a distance $\ell$ onto the plane of the detectors to determine the flux at a point $\left(x_{0}, y_{0}\right)$ on plane (3). All three planes are parallel as shown below, and we must have the radius of the pinhole $r_{p} \ll \ell_{0}$.

The plasma plane (1) has a finite thickness $d \ell$ so that the power radiating over $4 \pi$ steradians from the area $d S$ is $P(x, y)=p(x, y) d \ell d S$. Here, $p(x, y) W / m^{3}$ is the radiated power density at $(x, y)$. The flux on the pinhole on plane (2) from this power is $F_{p}=\frac{P(x, y)}{4 \pi r^{2}}\left(W / m^{2}\right)$. Now, the ray is at an angle $\alpha$ to the perpendicular, with $r \cos \theta=l$ and $r^{2}=l^{2}+x^{2}+y^{2}$, so that the power through the pinhole from $d S$ is $\mathrm{F}_{\mathrm{p}} \mathrm{S}_{\mathrm{p}} \cos \theta$. The pinhole is extremely small, so that the area dS maps geometrically into the area $\mathrm{dS}_{\mathrm{o}}$ and the coordinates map in a similar way; viz.,

$$
\ell^{2} \mathrm{dS}_{0}=\ell_{0}^{2} \mathrm{dS} \quad \text { and } \quad \ell x_{0}=-\ell_{0} x, \quad \ell y_{0}=\ell_{0} y
$$




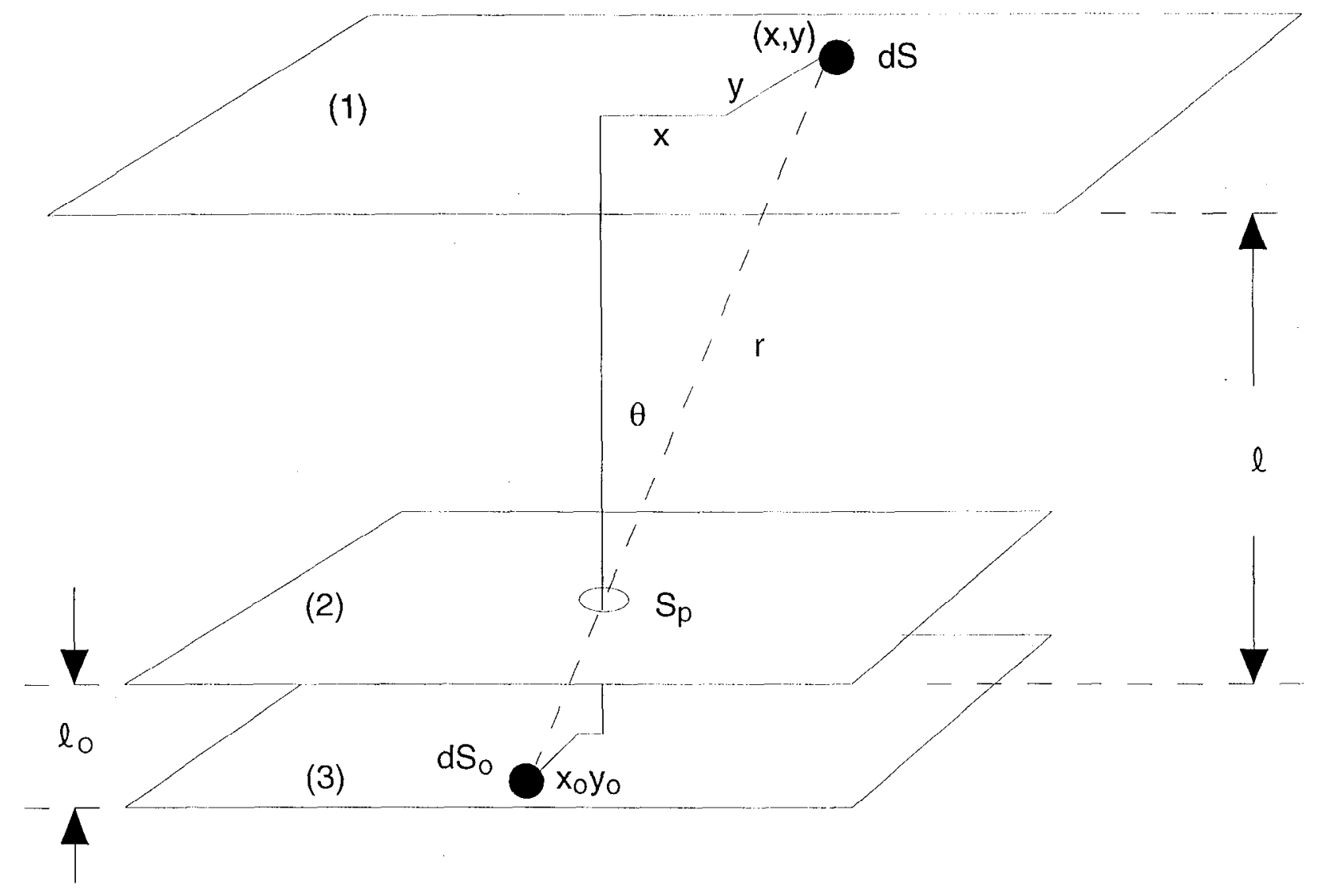

Power through the pinhole strikes the area $\mathrm{dS}_{0}$ on plane (3), so the flux perpendicular to plane (3) is $F\left(x_{0}, y_{0}\right)$, and the power on $d S_{0}$ is $P\left(x_{0}, y_{0}\right)$,

$$
P\left(x_{0}, y_{0}\right)=F\left(x_{0}, y_{0}\right) d S_{o}=F_{p} S_{p} \cos \theta=\frac{p(x, y) d l d S}{4 \pi r^{2}} S_{p} \cos \alpha
$$

and using $\ell^{2} \mathrm{dS}_{0}=\ell_{0}^{2} \mathrm{dS}$,

$$
F\left(x_{0}, y_{0}\right)=\frac{l^{2}}{l_{0}^{2}} \frac{p(x, y) d \ell}{4 \pi r^{2}} S_{p} \cos \theta \quad W / m^{2}
$$

(Note that the flux bundle passing through the pinhole is elliptical in shape in a plane perpendicular to the flux, owing to the angle $\alpha$, but the projection onto plane (3) is a circle).

If each detector surface is masked by a hole of area $S_{\text {det }}$ then the power to that detector is simply $\mathrm{dP}_{\mathrm{det}}=\mathrm{F}\left(\mathrm{x}_{0}, \mathrm{y}_{0}\right) \mathrm{S}_{\text {det }}$. So finally,

$$
\mathrm{dP} \text { det }=\frac{\ell^{2}}{\ell_{0}^{2}} \frac{[p(x, y) d \ell] S_{\text {det }}}{4 \pi r^{2}} S_{p} \cos \theta \quad \text { Watts }
$$

The 16 detectors in SSPX are distinguished only by their position $x_{0, n}$ or, equivalently, by their ray angle $\theta_{n}$ to the plasma region they sample.

Lastly, suppose we want to take a spherical surface through the plasma rather than the planar surface (1) shown above. This surface is a distance $r$ from the pinhole, and its thickness is dr. We now take an element $d S_{1}$ at plane (3) but coplanar with the element dS (both are perpendicular to the ray path). The area $\mathrm{dS}_{1}$ 
projects to $\mathrm{dS}_{\circ}$ (which is in plane (3) at a distance $r_{0}$ from the pinhole) through $d S_{0} \cos \theta=d S_{1}$. We follow the same proceedure above, except that the mapping is $r^{2} d_{0} \cos 0=r_{0}^{2} d S$.

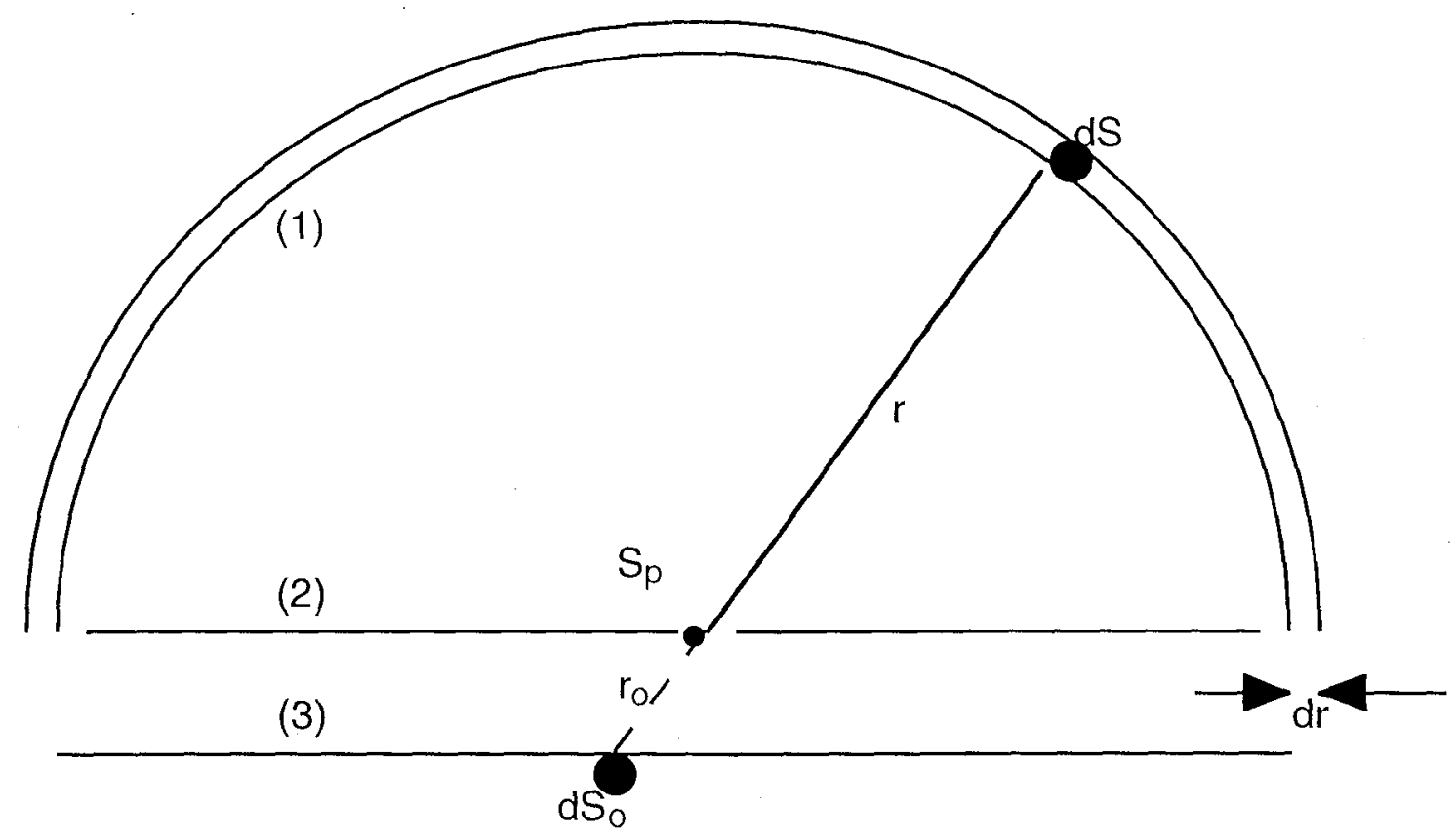

The flux from the plasma volume element incident on the pinhole is

$$
F_{p}=\frac{p(r) d r d S}{4 \pi r^{2}}
$$

and the amount of power passing through the pinhole is

$$
d P\left(r_{0}\right)=F_{p} S_{p} \cos \theta
$$

This power is incident on the area $\mathrm{dS}_{0}$ so there is a flux perpendicular to the detector plane of $F_{1}$, where

$$
F_{\perp}=\frac{d P}{d S_{0}}=\frac{p(r) d r}{4 \pi r_{0}} \frac{d r}{2} S_{p} \cos ^{2} \theta
$$

The power to the detector is $\mathrm{dP}_{\text {det }}=\mathrm{F}_{\perp} S_{\text {det }}$ and is independent of $r$. To get the total power along the plasma path length we integrate over $r$. For bolometry, where the plasma is divided into zones, the integral is replaced by sums of the power in each zone times length through that zone. Labeling the zones by $m=1-16$ the power on the $i^{\text {th }}$ detector from the $i^{\text {th }}$ ray is 


$$
\left(P_{\text {det }}\right)_{i}=\frac{S_{\text {det }} S_{p} \cos ^{2} \theta_{i}}{4 \pi\left(r_{o i}{ }^{2}\right)} \sum_{m=1}^{16} p_{m} \ell_{i-m}
$$

The area $S_{\text {det }}=493.4^{-1} \mathrm{~cm}^{2}$, defining $c=r_{o i} \cos \theta_{i}=(L+0.02)$ inches, so $c=2.92 \mathrm{~cm}$ gives $\frac{4 \pi c^{2}}{S_{p}}=33,856$. Defining a flux $F$,

$$
F_{i}=\frac{4 \pi c^{2}}{S_{p}} \frac{\left(P_{\text {det }}\right)_{i}}{S_{\operatorname{det} \cos ^{4} \theta_{i}}}=\ell_{i, m} P_{m}
$$

\section{Power calculations}

If we let $\mathbf{p}$ be a column vector for the radiation power densities in the different zones, and $\mathbf{F}$ be a column vector as defined above, then the above formula can be written in matrix form.

$$
F=L \bullet p \quad \text { and } \quad p=L^{-1} \bullet F
$$

If $\mathrm{p}_{\mathrm{m}}$ is in $\mathrm{W} / \mathrm{cm}^{3}$ and the lengths are in $\mathrm{cm}$, then $\mathrm{F}$ is in $\mathrm{W} / \mathrm{cm}^{2}$ when the detector area is given in $\mathrm{cm}^{2}$ then

$$
F_{i}=1.67 \times 10^{7} \frac{\left(P_{\text {det }}\right)_{i}}{\cos ^{4} \theta_{i}} \quad W / \mathrm{cm}^{2}
$$

The angles $\theta_{i}$ and the factors $\cos ^{-4}\left(\theta_{i}\right)$ for each ray are given in Table 1. Recall that the detector power is given by $V=13.65 P_{\text {det }}$ so that

Then

$$
F_{i}=1.224 \times 10^{6}\left(V_{i} \cos ^{-4} \theta_{i}\right) \quad W / \mathrm{cm}^{2}
$$

$$
\left.\mathrm{p}_{\mathrm{m}}=1.224\left[\mathrm{~L}^{-1}\right]_{\mathrm{i}, \mathrm{m}} \mathrm{V}_{\mathrm{i}} \cos ^{-4} \theta_{i}\right\}_{\mathrm{m}} \quad \mathrm{MW} / \mathrm{cm}^{3}
$$

Here, the detector voltages $V_{i}$ must be multiplied by $\cos ^{-4} \theta_{i}$, their corresponding enhancements that make up for the area losses from their slant angles to the perpendicular. Also, the power density above is now in $\mathrm{MW} / \mathrm{cm}^{3}$.

In the length matrix, columns are the zones, and rows are the lengths in the zone. Inversion can be done with Mathematica, where the column vectors such as $\mathbf{p}, \mathbf{F}$, and $\mathbf{V} \bullet \cos ^{-4} \theta$ are written as lists such as

$$
\begin{aligned}
& \mathbf{p}=\left\{p_{1}, p_{2}, p_{3}, p_{4}, \cdots \cdots\right\} \\
& v \cdot \cos ^{-4} \theta=\left\{V_{1} \cos ^{-4} \theta_{1}, V_{2} \cos ^{-4} \theta_{2}, V_{3} \cos ^{-4} \theta_{3}, V_{4} \cos ^{-4} \theta_{4}, \cdots \cdots\right\}
\end{aligned}
$$

Matricies are defined by lists of lists,

$$
L=\left\{\left\{L_{11}, L_{12}, L_{13} \bullet \bullet\right\},\left\{L_{21}, L_{22}, L_{23} \bullet \bullet,\left\{L_{31}, L_{32}, L_{33} \bullet \bullet, \bullet \bullet\right\}\right.\right.
$$


The operation we perform in Mathematica is $\mathbf{p}=$ Inverse[L].F, so we need the inverse of the length matrix. That matrix, multiplied 100-fold, is given in Table 4. Each element in the EXCEL spreadsheet carries 6 place accuracy. It was verified that the product of $L_{\bullet} \mathrm{L}^{-1}$ is a diagonal matrix, with off-diagonal values of the order of $10^{-17}$.

Table 4

Inverse length matrix (multiplied by 100) for the SSPX bolometer system

$\begin{array}{rrrrrrrrrrrrrrrr}29.8 & -26 & 0.68 & -1.5 & -0.5 & -0.4 & -0.2 & -0.2 & -0.1 & -0.1 & -0.1 & -0.1 & -0 & -0 & -0 & -0 \\ 0 & 12.9 & -10 & -0 & -0.8 & -0.3 & -0.3 & -0.2 & -0.1 & -0.1 & -0.1 & -0.1 & -0 & -0 & -0 & -0 \\ 0 & 0 & 9.33 & -7.1 & 0.04 & -0.6 & -0.3 & -0.2 & -0.1 & -0.1 & -0.1 & -0.1 & -0 & -0 & -0 & -0 \\ 0 & 0 & 0 & 7.57 & -5.7 & 0.08 & -0.5 & -0.2 & -0.2 & -0.1 & -0.1 & -0.1 & -0.1 & -0 & -0 & -0 \\ 0 & 0 & 0 & 0 & 6.51 & -4.9 & 0.11 & -0.4 & -0.2 & -0.1 & -0.1 & -0.1 & -0.1 & -0 & -0 & -0 \\ 0 & 0 & 0 & 0 & 0 & 5.81 & -4.3 & 0.13 & -0.4 & -0.1 & -0.1 & -0.1 & -0.1 & -0 & -0 & -0 \\ 0 & 0 & 0 & 0 & 0 & 0 & 5.34 & -4 & 0.15 & -0.4 & -0.1 & -0.1 & -0.1 & -0.1 & -0 & -0 \\ 0 & 0 & 0 & 0 & 0 & 0 & 0 & 5.02 & -3.7 & 0.17 & -0.3 & -0.1 & -0.1 & -0.1 & -0.1 & -0 \\ 0 & 0 & 0 & 0 & 0 & 0 & 0 & 0 & 4.82 & -3.6 & 0.18 & -0.3 & -0.1 & -0.1 & -0.1 & -0 \\ 0 & 0 & 0 & 0 & 0 & 0 & 0 & 0 & 0 & 4.71 & -3.5 & 0.2 & -0.3 & -0.1 & -0.1 & -0.1 \\ 0 & 0 & 0 & 0 & 0 & 0 & 0 & 0 & 0 & 0 & 4.68 & -3.5 & 0.22 & -0.3 & -0.1 & -0.1 \\ 0 & 0 & 0 & 0 & 0 & 0 & 0 & 0 & 0 & 0 & 0 & 4.71 & -3.5 & 0.24 & -0.3 & -0.1 \\ 0 & 0 & 0 & 0 & 0 & 0 & 0 & 0 & 0 & 0 & 0 & 0 & 4.81 & -3.6 & 0.26 & -0.3 \\ 0 & 0 & 0 & 0 & 0 & 0 & 0 & 0 & 0 & 0 & 0 & 0 & 0 & 4.97 & -3.7 & 0.26 \\ 0 & 0 & 0 & 0 & 0 & 0 & 0 & 0 & 0 & 0 & 0 & 0 & 0 & 0 & 5.18 & -3.8 \\ 0 & 0 & 0 & 0 & 0 & 0 & 0 & 0 & 0 & 0 & 0 & 0 & 0 & 0 & 0 & 5.03\end{array}$

Note that the largest values of this matrix are positive and on the diagonal, and that all other values in each row are very small except the negative element just next to the diagonal. So, in adding the contribution from the different detectors to determine the power from a given zone the result is the following. For a zone, such as zone 5 , we multiply the elements in row 5 times the vector $\mathbf{F}$ which represent the signals from each of the 16 channels. There is no contribution from the first 4 zones of course, but by far the largest contributions to the value $100 p_{5}$ are $6.51 F_{5}-4.9 F_{6}$. In general, for zone $m$, the contributions are largely from the rays $i=m, m+1$ with the second contribution subtracting from the first. The negative values next to the diagonal are all very close to $75 \%$ of the diagonal value, except for rows one and two which are $89 \%$ and $78 \%$ respectively.

Summarizing, the first two inner zones have approximate values given by $100 p_{1} \approx 29.76 F_{1}-26.49 F_{2}$, and $100 p_{15} \approx 12.9 F_{2}-10.1 F_{3}$. Thereafter, the values are approximately $100 p_{m} \approx D_{m}\left(F_{m}-0.25 F_{m+1}\right)$, where $D_{m}$ is the diagonal element in the $\mathrm{m}^{\text {th }}$ column above. Of course, $100 \mathrm{p}_{16}=5.03 \mathrm{~F}_{16}$.

One way to look at this data reduction is that the outemost zone $(m=16)$ power density is determined directly by detector 16 , and all other zone powers are determined by subtracting about $3 / 4$ of the signal (except for the $\cos ^{-4} \theta$ correction) from the previous zone (working from outside to inside) to get its power density. It is commonly stated that the power for each zone is determined from bolometry by first 
computing the outermost value and working inward by subtraction, impying that all internal values are sensitive to the outer ones.

But a different interpretation, supported by the peculiarity of the inverse matrix above, is that the power for a given zone is simply determined by signals from the detector with that zone number $(i=m)$ and the adjacent one $(i=m+1)$, independent of all other signals if they are not more than about an order of magnitude larger than the two used for the particular zone.

To summarize the flux vector in $\mathrm{MW} / \mathrm{cm}^{2}$

$$
\begin{gathered}
F=1.224\left\{1.64 V_{1}, 1.47 V_{2}, 1.33 V_{3}, 1.21 V_{4}, 1.13 V_{5} 1.06 V_{6}, 1.02 V_{7}, V_{8},\right. \\
\left.V_{9}, 1.02 V_{10}, 1.06 V_{11}, 1.13 V_{12}, 1.21 V_{13}, 1.33 V_{14}, 1.47 V_{15}, 1.64 V_{16}\right\}
\end{gathered}
$$

Multiplying $\mathrm{F}$ by the inverse length matrix (in $\mathrm{cm}^{-1}$ ) gives the power densities in $\mathrm{MW} / \mathrm{cm}^{3}$. The total radiated power is estimated from $\sum_{m=1}^{16} p_{m} v_{m}$ where the volume elements for the $m$ zones are computed from the radii defining the zones in Table 2 . We find the values for the areas $A_{m}$ below in Table 5 . These are to be multiplied by some vertical height, $\approx 50 \mathrm{~cm}$, to get the volumes.

Table 5. Volume elements in the 16 zones

$\begin{array}{cc}\mathrm{m} & \begin{array}{c}\text { Area } \\ \mathrm{cm}^{\wedge} 2\end{array} \\ 1 & 8.868 \\ 2 & 73.71 \\ 3 & 157 \\ 4 & 248.6 \\ 5 & 344.9 \\ 6 & 440.8 \\ 7 & 530.8 \\ 8 & 609 \\ 9 & 670.3 \\ 10 & 711 \\ 11 & 729.7 \\ 12 & 726.9 \\ 13 & 705.1 \\ 14 & 667.8 \\ 15 & 619.4 \\ 16 & 610.1\end{array}$

\section{Acknowledgement}

Thanks to D. Ryutov for showing me the mapping technique for calculating the flux on the plane of the detectors. The bolometers were constructed by A. Ahmed and the SSPX technical staff. This work was performed under the auspices of the U.S. Department of Energy by the University of California, Lawrence Livermore National Laboratory, under contract No. W-7405-Eng-48. 